\title{
Nephrotic Syndrome with Lesion of Endothelial Glomerulonephritis
}

National Cancer Institute

\section{Source}

National Cancer Institute. Nephrotic Syndrome with Lesion of Endothelial

Glomerulonephritis. NCI Thesaurus. Code C35535.

A renal functional disorder characterized by proteinuria, edema, hyperlipidemia and hypoalbuminemia. It results from damage to the renal vascular filtration apparatus. It is further characterized by an inflammatory reaction in the endovascular lining of the glomerular capillaries. Sequelae may include hypertension, atherosclerosis, infection, hypercoagulablity and renal failure. 